\title{
Pathwise Stability of Degenerate Stochastic Evolutions
}

\author{
Joris Bierkens
}

\begin{abstract}
For linear stochastic evolution equations with linear multiplicative noise, a new method is presented for estimating the pathwise Lyapunov exponent. The method consists of finding a suitable (quadratic) Lyapunov function by means of solving an operator inequality. One of the appealing features of this approach is the possibility to show stabilizing effects of degenerate noise. The results are illustrated by applying them to the examples of a stochastic partial differential equation and a stochastic differential equation with delay. In the case of a stochastic delay differential equation our results improve upon earlier results.
\end{abstract}

Mathematics Subject Classification (2010). 60Hxx, 93Dxx, 47A62, $34 \mathrm{~K} 50$.

Keywords. Stochastic evolution equation, Lyapunov exponent, pathwise stability, stochastic delay differential equation, operator inequality.

\section{Introduction}

In this paper we discuss a new approach to the estimation of the pathwise Lyapunov exponent of linear stochastic evolution equations with multiplicative noise. From such an estimate pathwise stability may be deduced.

Stochastic evolution equations provide a general framework for describing complex systems (high- or infinite-dimensional) under random influence. There exists an extensive amount of literature on stochastic evolution equations, or stochastic differential equations in infinite dimensions; see for example $[15,21,32]$.

One of the primary ways to characterize dynamical systems qualitatively is to study the question of stability. For stochastic evolutions different notions of stability arise in a natural way: stability in $p$-th moment, stability in probability and pathwise or almost sure stability. It can be argued that 
from a practical point of view pathwise stability is most relevant, since sample paths represent the observed behaviour of systems modelled by stochastic evolution equations.

Important results on moment stability and pathwise stability of linear stochastic evolutions may be found in e.g. $[14,19,20]$. A survey of results on linear and non-linear stochastic evolution equations may be found in [27].

Stochastic evolutions with delay are our primary inspiration. For this special case results on stability are obtained in e.g. [1]. It is shown there that noise may have a stabilizing effect, a result not obtained in the references mentioned above on stability of general stochastic evolutions. In this paper we wish to obtain this stabilizing effect of noise without using the special structure of delay differential equations. Instead, we employ a general semigroup approach and use only dissipativeness of the semigroup and general estimates on the semigroup and the perturbation by noise.

Results of [23] for systems with commuting operators show that in case the noise is non-degenerate it may very well help in stabilizing the stochastic evolution. However in the case of stochastic delay equations, the noise can never influence the past, resulting in degeneracy of the noise. The result of [23] is improved upon in [11], but here one still depends on commutativity of operators. The generator of the delay semigroup and the noise operator are not commutative, such that results from [11] are still not applicable to stochastic delay differential equations.

We propose a different method, in which we use a quadratic Lyapunov function. As is well known to such a quadratic form corresponds a linear operator. We will rephrase the problem of finding a quadratic form which provides us with the best estimate on the Lyapunov exponent of a given stochastic evolution, as an operator inequality for the mentioned operator. Conditions for the solution of the operator inequality then result in conditions for stability of the stochastic evolution. Our results are applied to stochastic partial differential equations, as a simple illustration, and to stochastic delay differential equations, for which a new stability result is obtained.

\section{Preliminary Results}

Let $H$ be a real Hilbert space, and consider the linear SDE in mild form

$$
X(t)=S(t) x+\sum_{i=1}^{k} \int_{0}^{t} S(t-s) B_{i} X(s) d W_{i}(s),
$$

where $W_{i}, i=1, \ldots, k$, are independent standard Brownian motions in $\mathbb{R},(S(t))_{t \geq 0}$ is a strongly continuous semigroup on $H$ with generator $A$ : $\mathfrak{D}(A) \rightarrow \bar{H}, B_{i} \in L(H), i=1, \ldots, k$ and $x \in H$. A solution to such an equation always exists and is unique (see [15]). Note that (1) is the variation of constants formulation of

$$
\left\{\begin{array}{l}
d X(t)=A X(t) d t+\sum_{i=1}^{k} B_{i} X(t) d W_{i}(t), \quad t \geq 0 \\
X(0)=x
\end{array}\right.
$$


Define the Lyapunov exponent as the random variable

$$
\lambda:=\limsup _{t \rightarrow \infty} \frac{1}{t} \log |X(t ; x)|, \quad \text { a.s. }
$$

with $X(\cdot ; x)$ the solution of $(1)$.

We say that the stochastic evolution described by (1) is pathwise asymptotically stable if

$$
\lim _{t \rightarrow \infty}|X(t ; x)|=0, \quad \text { almost surely. }
$$

We have the following relation between the Lyapunov exponent and the notion of pathwise stability.

Proposition 2.1. If $\lambda<0$ almost surely then (1) describes a pathwise asymptotically stable evolution.

Proof. Suppose $\limsup _{t \rightarrow \infty}|X(t ; x)|>\varepsilon$ on some $\mathcal{F}$-measurable $E \subset \Omega$. Then

$$
0 \leq \leq \limsup _{t \rightarrow \infty} \frac{1}{t} \log |X(t ; x)| \leq \lambda<0 \quad \text { on } E,
$$

a contradiction.

Note that $\lambda \leq 0$ is not sufficient: consider the deterministic evolution described by

$$
\dot{x}(t)=\left(\begin{array}{ll}
0 & 0 \\
1 & 0
\end{array}\right) x(t), \quad x(0)=\left(\begin{array}{l}
1 \\
0
\end{array}\right)
$$

with solution

$$
x(t)=\left(\begin{array}{l}
1 \\
t
\end{array}\right) .
$$

The Lyapunov exponent of $(x(t))_{t \geq 0}$ is 0 but the evolution is clearly not asymptotically stable.

We recall the following notions concerning linear operators and strongly continuous semigroups. Let $A$ be a closed linear operator and let $\rho(A)$ denote the resolvent set of $A$ :

$$
\rho(A)=\left\{\lambda \in \mathbb{C}:(\lambda-A)^{-1} \text { exists and is bounded }\right\} .
$$

Define the spectrum $\sigma(A)$ of $A$ by $\sigma(A)=\mathbb{C} \backslash \rho(A)$. Let $\mathfrak{s}(A)$ denote the spectral bound of $A$, and $\mathfrak{r}(B)$ the spectral radius of $B$, i.e.

$\mathfrak{s}(A):=\sup \{\operatorname{Re} \lambda: \lambda \in \sigma(A)\} \quad$ and $\quad \mathfrak{r}(B):=\sup \{|\lambda|: \lambda \in \sigma(B)\}$.

Furthermore let $\omega_{0}(A)$ denote the growth bound of $A$, i.e.

$\omega_{0}(A):=\inf \left\{\omega \in \mathbb{R}: \exists_{M \geq 1}\right.$ s.t. $\|\exp (A t)\| \leq M \mathrm{e}^{\omega t}$ for all $\left.t \geq 0\right\}$.

Proposition 2.2. We have the following relation between $\mathfrak{s}(A), \omega_{0}(A)$ and $\mathfrak{r}(S(t))$ :

$$
\mathfrak{s}(A) \leq \omega_{0}(A)=\frac{1}{t} \log \mathfrak{r}(S(t)), \quad \text { for all } t \geq 0 .
$$

If $(S(t))_{t \geq 0}$ is eventually norm continuous then the inequality in (3) becomes an equality: $\mathfrak{s}(A)=\omega_{0}(A)$. 
See [18], Proposition IV.2.2, and Theorem IV.3.11. Important cases where $(S(t))$ is eventually norm continuous are the case where $H$ is finite dimensional, and where the semigroup $(S(t))$ is (eventually) compact or analytic.

Now we consider the particular case of (1) where all operators commute. The following result is slightly sharper and more general than a result on commutative systems found in [23]. A stronger version, allowing for unbounded operators $B_{i}$, may be found in [11].

Proposition 2.3. Suppose $A$ and all $B_{i}, i=1, \ldots, k$ commute. Then

$$
\limsup _{t \rightarrow \infty} \frac{1}{t} \log |X(t ; x)| \leq \omega_{0}\left(A-\frac{1}{2} \sum_{i=1}^{k} B_{i}^{2}\right), \quad \text { a.s. }
$$

Proof. Let $\left(T_{0}\right)_{t \geq 0}$ be the strongly continuous semigroup generated by $A-\frac{1}{2} \sum_{i=1}^{k} B_{i}^{2}$, and let $\left(T_{i}\right)_{t \geq 0}$ be the uniformly continuous groups $\left(T_{i}(t)\right)_{t \geq 0}$ generated by $B_{i}, i=1, \ldots, k$.

Note that the solution is given by

$$
X(t ; x)=T_{0}(t) \prod_{i=1}^{k} T_{i}\left(W_{i}(t)\right) x . \quad t \geq 0, x \in H .
$$

Suppose $\left\|T_{i}(t)\right\| \leq M_{i} \exp \left(\omega_{0}\left(B_{i}\right) t\right), i=1, \ldots, k$. Then

$$
\frac{1}{t} \log |X(t ; x)| \leq \frac{1}{t} \log |x|+\frac{1}{t} \log \left\|T_{0}(t)\right\|+\frac{1}{t} \sum_{i=1}^{k} \log \left\|T_{i}\left(W_{i}(t)\right)\right\| .
$$

Now using the strong law of large numbers for martingales (see Theorem A.1)

$$
\limsup _{t \rightarrow \infty} \frac{1}{t} \log \left\|T_{i}\left(W_{i}(t)\right)\right\| \leq \limsup _{t \rightarrow \infty} \frac{\log M_{i}}{t}+\frac{\omega_{0}\left(B_{i}\right)\left|W_{i}(t)\right|}{t}=0 \quad \text { a.s. }
$$

and furthermore

$$
\limsup _{t \rightarrow \infty} \frac{1}{t} \log \left\|T_{0}(t)\right\|=\omega_{0}\left(A-\frac{1}{2} \sum_{i=1}^{k} B_{i}^{2}\right) .
$$

The stated result is now obtained by combining the above estimates in (5).

It may be seen from this proposition that in the commutative case, noise may have a stabilizing effect even when it is degenerate:

Example 2.4. Let $A=\left(\begin{array}{cc}-1 & 0 \\ 0 & 1\end{array}\right)$ and $B=\left(\begin{array}{ll}0 & 0 \\ 0 & 2\end{array}\right)$. Then $A-\frac{1}{2} B^{2}=$ $\left(\begin{array}{cc}-1 & 0 \\ 0 & -1\end{array}\right)$

In the remainder of this paper we will establish a stabilizing effect of degenerate noise in the non-commutative case. 


\section{Bounded Case}

First we consider the case where $A \in L(H)$, so that $(S(t))_{t \geq 0}$ is a uniformly continuous semigroup, in which case we may apply Itô's formula.

Recall the notion of a coercive operator.

Definition 3.1. An operator $T \in L(H)$ is called coercive if $\langle T x, x\rangle \geq \gamma|x|^{2}$ for all $x \in H$ and some $\gamma>0$.

Lemma 3.2. Suppose there exists a self-adjoint coercive operator $Q \in L(H)$ and $\lambda \in \mathbb{R}$ such that, for all $x \in H$,

$$
\langle Q x, x\rangle\left[2\langle Q A x, x\rangle+\sum_{i=1}^{k}\left\langle Q B_{i} x, B_{i} x\right\rangle-2 \lambda\langle Q x, x\rangle\right] \leq 2 \sum_{i=1}^{k}\left\langle Q x, B_{i} x\right\rangle^{2} .
$$

Let $Y(t):=\langle Q X(t), X(t)\rangle$, with $X$ the solution of $(1)$.

Then

$$
\limsup _{t \rightarrow \infty} \frac{1}{t} \log Y(t) \leq 2 \lambda, \quad \text { a.s. }
$$

Proof. If $x=0$, then $\mathbb{P}(X(t)=0)=1, t \geq 0$, and the required estimate holds trivially.

Suppose $x \neq 0$. Then by uniqueness of the solution of SDEs and positiveness of $Q, \mathbb{P}(Y(t)=0)=0$ for all $t \geq 0$.

By Itô's formula,

$$
\begin{aligned}
d \log Y(t)= & \left\{\frac{1}{Y(t)}\left[2\langle Q A X(t), X(t)\rangle+\sum_{i=1}^{k}\left\langle Q B_{i} X(t), B_{i} X(t)\right\rangle\right]\right. \\
& \left.-\frac{2}{Y(t)^{2}} \sum_{i=1}^{k}\left\langle Q X(t), B_{i} X(t)\right\rangle^{2}\right\} d t \\
& +\frac{2}{Y(t)} \sum_{i=1}^{k}\left\langle Q x(t), B_{i} X(t)\right\rangle d W_{i}(t) \\
\leq & 2 \lambda d t+\frac{2}{Y(t)} \sum_{i=1}^{k}\left\langle Q X(t), B_{i} X(t)\right\rangle d W_{i}(t) .
\end{aligned}
$$

Now by boundedness of $\frac{\left\langle Q X(t), B_{i} X(t)\right\rangle}{\langle Q X(t), X(t)\rangle}$ for $i=1, \ldots, k$ and the law of large numbers for martingales (Theorem A.1)

$$
\frac{1}{t} \int_{0}^{t} \frac{2\left\langle Q X(s), B_{i} X(s)\right\rangle}{Y(s)} d W_{i}(s) \rightarrow 0 \quad(t \rightarrow \infty), \quad \text { a.s. }, \quad i=1, \ldots, k,
$$

so

$$
\limsup _{t \rightarrow \infty} \frac{1}{t} \log Y(t) \leq 2 \lambda \quad \text { a.s. }
$$


Proposition 3.3. Suppose there exists a self-adjoint operator $Q \in L(H), \lambda \in \mathbb{R}$ and $b_{i} \in \mathbb{R}, i=1, \ldots, k$ such that

$$
\left(A+\sum_{i=1}^{k} b_{i} B_{i}\right)^{*} Q+Q\left(A+\sum_{i=1}^{k} b_{i} B_{i}\right)+\sum_{i=1}^{k} B_{i}^{*} Q B_{i}+\left(\frac{1}{2} \sum_{i=1}^{k} b_{i}^{2}-2 \lambda\right)
$$

is negative semidefinite.

Then, with $Y$ as in Lemma 3.2,

$$
\limsup _{t \rightarrow \infty} \frac{1}{t} \log Y(t) \leq 2 \lambda, \quad \text { a.s. }
$$

Proof. Note that (by the $a b c$-formula), for $i=1, \ldots, k$,

$$
\frac{\left\langle Q x, B_{i} x\right\rangle^{2}}{\langle Q x, x\rangle^{2}}+b_{i} \frac{\left\langle Q x, B_{i} x\right\rangle}{\langle Q x, x\rangle}+\frac{1}{4} b_{i}^{2} \geq 0, \text { for all } x \in H .
$$

So, by Lemma 3.2 , if

$$
\begin{aligned}
& 2\langle Q A x, x\rangle+\sum_{i=1}^{k}\left\langle Q B_{i} x, B_{i} x\right\rangle-2 \lambda\langle Q x, x\rangle \\
& \quad \leq-2 \sum_{i=1}^{k} b_{i}\left\langle Q x, B_{i} x\right\rangle-\frac{1}{2} \sum_{i=1}^{k} b_{i}^{2}\langle Q x, x\rangle \text { for all } x \in H,
\end{aligned}
$$

then the claimed result holds.

But this is equivalent to the stated condition.

The following theorem gives a sufficient condition in order for a solution to (6) to exist.

Theorem 3.4. Suppose $D_{j} \in L(H), j=1, \ldots, k, L$ is the generator of $a$ strongly continuous semigroup $(T(t))_{t \geq 0}$ acting on $H$ such that

$$
\|T(t)\| \leq m \mathrm{e}^{\omega t} \quad \text { for all } t \geq 0,
$$

with $m \geq 1, \omega \in \mathbb{R}$, and

$$
m^{2} \sum_{j=1}^{k}\left\|D_{j}\right\|^{2}+2 \omega<0
$$

Then for any $M \in L(H)$ there exists a unique solution $Q \in L(H)$ to the equation

$$
L^{*} Q+Q L+\sum_{j=1}^{k} D_{j}^{*} Q D_{j}=M
$$

which should be interpreted as

$$
\langle Q x, L y\rangle+\langle Q L x, y\rangle+\sum_{j=1}^{k}\left\langle Q D_{j} x, D_{j} y\right\rangle=\langle M x, y\rangle \quad \text { for all } x, y \in \mathfrak{D}(L) \text {. }
$$


This $Q$ also satisfies

$$
Q=\int_{0}^{\infty} T^{*}(t)\left(\sum_{j=1}^{k} D_{j}^{*} Q D_{j}-M\right) T(t) d t .
$$

We can estimate the norm of $Q$ by

$$
\|Q\| \leq-\frac{\|M\| m^{2}}{2 \omega+m^{2} \sum_{j=1}^{k}\left\|D_{j}\right\|^{2}} .
$$

Furthermore,

(i) if $M=0$ then $Q=0$,

(ii) if $M \leq 0$ then $Q \geq 0$, and

(iii) if $M<0$ then $Q>0$.

Proof. Define a recursion by

$$
Q_{0}:=0, \quad Q_{i+1}:=\int_{0}^{\infty} T^{*}(t)\left(\sum_{j=1}^{k} D_{j}^{*} Q_{i} D_{j}-M\right) T(t) d t .
$$

The recursion is actually a contraction, since

$$
\begin{aligned}
\left\|Q_{i+1}-Q_{i}\right\| & =\left\|\sum_{j=1}^{k} \int_{0}^{\infty} T^{*}(t) D_{j}^{*}\left(Q_{i}-Q_{i-1}\right) D_{j} T(t) d t\right\| \\
& \leq m^{2} \sum_{j=1}^{k}\left\|D_{j}\right\|^{2} \int_{0}^{\infty} \mathrm{e}^{2 \omega t} d t\left\|Q_{i}-Q_{i-1}\right\| \\
& =-\frac{m^{2} \sum_{j=1}^{k}\left\|D_{j}\right\|^{2}}{2 \omega}\left\|Q_{i}-Q_{i-1}\right\| .
\end{aligned}
$$

Note that the recursion is defined such that $Q_{i+1}$ satisfies

$$
L^{*} Q_{i+1}+Q_{i+1} L=M-\sum_{j=1}^{k} D_{j}^{*} Q_{i} D_{j},
$$

a basic result from Lyapunov theory (see [13, Theorem 4.1.23]).

Hence there exists a unique fixed point $Q \in L(H)$ that satisfies both (9) and

$$
Q=\int_{0}^{\infty} T^{*}(t)\left(\sum_{j=1}^{k} D_{j}^{*} Q D_{j}-M\right) T(t) d t .
$$

Hence

$$
\begin{aligned}
\|Q\| & \leq \int_{0}^{\infty} m^{2} \mathrm{e}^{2 \omega t}\left(\sum_{j=1}^{k}\left\|D_{j}\right\|^{2}\|Q\|+\|M\|\right) d t \\
& =\frac{m^{2}}{-2 \omega}\left(\sum_{j=1}^{k}\left\|D_{j}\right\|^{2}\|Q\|+\|M\|\right) .
\end{aligned}
$$


By repeating this estimate $n$ times, we obtain

$$
\|Q\| \leq\left(\frac{m^{2} \sum_{j=1}^{k}\left\|D_{j}\right\|^{2}}{-2 \omega}\right)^{n}\|Q\|+\frac{m^{2}\|M\|}{-2 \omega} \sum_{i=0}^{n-1}\left(\frac{m^{2} \sum_{j=1}^{k}\left\|D_{j}\right\|^{2}}{-2 \omega}\right)^{i} .
$$

Let $n \rightarrow \infty$ to obtain

$$
\|Q\| \leq \frac{m^{2}\|M\|}{-2 \omega} \frac{1}{1-\frac{m^{2} \sum_{j=1}^{k}\left\|D_{j}\right\|^{2}}{-2 \omega}}=-\frac{m^{2}\|M\|}{m^{2} \sum_{j=1}^{k}\left\|D_{j}\right\|^{2}+2 \omega} .
$$

If $M=0$ then $Q=0$ by uniqueness of the solution.

Now suppose $M \leq 0$. Then we can check that the recursion for $\left(Q_{i}\right)$ has the property that $Q_{i} \geq 0$ for all $i$. So $Q \geq 0$, and (11) shows that

$$
Q \geq-\int_{0}^{\infty} T^{*}(t) M T(t) d t
$$

If $M<0$, then there exists a unique $P \in L(H), P>0$ such that $M=L^{*} P+P L$. Then

$$
Q \geq-\int_{0}^{\infty} T^{*}(t) M T(t) d t=P>0
$$

So far we only know that if $Q \in L(H)$ a solution to (9) with $M<0$, then $Q>0$. But to obtain equivalence of norms we need $Q$ to be coercive. In the finite-dimensional case coerciveness of $Q$ is implied by $Q>0$ but in infinite dimensions this is not the case. The next proposition shows that we can find a coercive solution in case $L$ is dissipative, or equivalently if $(T(t))_{t \geq 0}$ is a contraction semigroup.

Proposition 3.5. Suppose $L,\left(D_{j}\right)_{j=1, \ldots, k}$ are as in Proposition 3.4 and that $(8)$ holds. Then there exists a $Q \in L(H)$ such that (10) holds with $M=L+L^{*}$. Furthermore for this $Q$ we have $Q \geq I$ and

$$
\|Q\| \leq \frac{-2 \omega}{-\left(2 \omega+m^{2} \sum_{j=1}^{k}\left\|D_{j}\right\|^{2}\right)} .
$$

Note that if $L$ is dissipative, then $\langle M x, x\rangle=2\langle L x, x\rangle \leq 0$ for all $x \in H$. Proof. By Proposition 3.4, there exists a unique solution $R \in L(H), R \geq 0$ to (10) with $M=-\sum_{j=1}^{k} D_{j}^{*} D_{j}$. Furthermore $R \geq 0$ and, by (12)

$$
\|R\| \leq \frac{\sum_{j=1}^{k}\left\|D_{j}\right\|^{2} m^{2}}{-\left(2 \omega+m^{2} \sum_{j=1}^{k}\left\|D_{j}\right\|^{2}\right)}
$$

Let $Q:=I+R$. Then $Q \geq I$, the claimed estimate for $Q$ holds and

$$
L^{*} Q+Q L+\sum_{j=1}^{k} D_{j}^{*} Q D_{j}=L^{*}+L+\sum_{j=1}^{k} D_{j}^{*} D_{j}-\sum_{j=1}^{k} D_{j}^{*} D_{j}=L^{*}+L,
$$

interpreted in the weak sense of (10). 


\section{Unbounded Case}

We will now extend the result of the previous section to the case where $A$ is possibly unbounded and does no longer generate a uniformly continuous semigroup. We do this by employing approximations of $A$ by bounded operators $\left(A_{n}\right)$. The background for this technique is discussed in Appendix B.

Recall that $H$ is a real Hilbert space, and we consider the linear SDE in mild form

$$
X(t)=S(t) x+\int_{0}^{t} S(t-s) B X(s) d W(s)
$$

where $W$ is a standard Brownian motion in $\mathbb{R},(S(t))_{t \geq 0}$ is a strongly continuous semigroup on $H$ with generator $A: \mathfrak{D}(A) \rightarrow H, B \in L(H)$ and $x \in H$.

Remark 4.1. From this point onward we assume for notational convenience $k=1$, i.e. the stochastic process $X$ is driven by only one standard Brownian motion. There is however no problem in proving all the results of this section for the case with multiple Brownian motions.

Lemma 4.2. Suppose $T=(T(t))_{t \geq 0}$ is a strongly continuous semigroup with approximation $\left(T_{n}\right)_{n \in \mathbb{N}}$ such that, for some $\omega<0$ and $m \geq 1$,

$$
\|T(t)\| \vee \sup _{n \in \mathbb{N}}\left\|T_{n}(t)\right\| \leq m \mathrm{e}^{\omega t} .
$$

Let $L$ and $L_{n}$ denote the generators of $(T(t))_{t>0}$ and $\left(T_{n}(t)\right)_{t>0}, n \in \mathbb{N}$, respectively. For $n \in \mathbb{N}$ let $R_{n} \geq 0$ denote the unique positive semidefinite solution in $L(H)$ to the Lyapunov equation

$$
L_{n}^{*} R_{n}+R_{n} L_{n}=M
$$

for some fixed $M \in L(H), M \leq 0$.

Then $\left\langle y, R_{n} x\right\rangle \rightarrow\langle y, R x\rangle$ for any $x, y \in H$, where $R \geq 0$ is the unique positive semidefinite solution in $L(H)$ to

$$
L^{*} R+R L=M \text {. }
$$

Proof. We have, using the representation (see [13, Theorem 4.1.23])

$$
R=-\int_{0}^{\infty} T(t)^{*} M T(t) d t, \quad R_{n}=-\int_{0}^{\infty} T_{n}(t)^{*} M T_{n}(t) d t, \quad n \in \mathbb{N},
$$

that for any $t>0$,

$$
\begin{aligned}
&\left|\left\langle y, R_{n} x-R x\right\rangle\right| \\
&=\left|\left\langle y, \int_{0}^{\infty} T_{n}^{*}(s) M T_{n}(s) x-T^{*}(s) M T(s) x d s\right\rangle\right| \\
&=\left|\int_{0}^{\infty}\left\langle T_{n}(s) y, M\left(T_{n}(s)-T(s)\right) x\right\rangle+\left\langle\left(T_{n}(s)-T(s)\right) y, M T(s) x\right\rangle d s\right|
\end{aligned}
$$




$$
\begin{aligned}
& \leq \int_{0}^{\infty}\left\|T_{n}(s)\right\|\|y\|\|M\|\left|\left(T_{n}(s)-T(s)\right) x\right| d s \\
& \quad+\int_{0}^{\infty}\left|\left(T_{n}(s)-T(s)\right) y\right|\|M\|\|T(s)\|\|x\| d s \\
& \leq m\|M\| \int_{0}^{\infty} \mathrm{e}^{\omega s}\left(\|y\|\left|\left(T_{n}(s)-T(s)\right) x\right|+\left|\left(T_{n}(s)-T(s)\right) y\right|\|x\|\right) d s .
\end{aligned}
$$

For the first term we have

$$
\int_{0}^{\infty} \mathrm{e}^{\omega s}\left|\left(T_{n}(s)-T(s)\right) x\right| \leq \int_{0}^{t} \mathrm{e}^{\omega s}\left|\left(T_{n}(s)-T(s)\right) x\right| d s+2 m|x| \int_{t}^{\infty} \mathrm{e}^{2 \omega s} d s .
$$

Now pick $t$ large enough such that the second term is smaller than $\varepsilon / 2$. Since $\left(T_{n}\right)$ is an approximation of $T$, we have uniform convergence in $s \in[0, t]$ of $\left|T_{n}(s) x-T(s) x\right|$. So let $N$ large enough such that $\left|T_{n}(s) x-T(s) x\right| \leq \delta$ for all $s \in[0, t]$ and for $\delta>0$ such that

$$
\int_{0}^{t} \mathrm{e}^{\omega s} \delta d s<\varepsilon / 2
$$

Repeating this argument for the second term leads to the stated result.

Lemma 4.3. Suppose $(T(t))_{t \geq 0}$ is a strongly continuous semigroup with approximation $\left(T_{n}\right)_{n \in \mathbb{N}}$ and infinitesemal generators $L$ and $\left(L_{n}\right)_{n \in \mathbb{N}}$, respectively. Suppose that for some $m \geq 1$ and $\omega<0$ we have

$$
\|T(t)\| \vee \sup _{n \in \mathbb{N}}\left\|T_{n}(t)\right\| \leq m \mathrm{e}^{\omega t},
$$

and suppose for this $m, \omega$ and some $D \in L(H)$ condition (8) holds.

Let $M \in L(H)$ be self-adjoint and negative semidefinite. Let $Q$ and $Q^{n}$, $n \in \mathbb{N}$, denote the unique positive semidefinite solutions to

$$
L^{*} Q+Q L+D^{*} Q D=M \quad \text { and } L_{n}^{*} Q^{n}+Q^{n} L_{n}+D^{*} Q^{n} D=M .
$$

Then for all $x, y \in H$ we have that $\left\langle x, Q^{n} y\right\rangle \rightarrow\langle x, Q y\rangle$ as $n \rightarrow \infty$.

Proof. For $n \in \mathbb{N}$ construct a recursion by

$$
Q_{0}^{n}:=0 \quad \text { and } \quad Q_{j+1}^{n}:=\int_{0}^{\infty} T_{n}^{*}(t)\left(D^{*} Q_{j}^{n} D-M\right) T_{n}(t) d t, \quad j \in \mathbb{N} .
$$

Similarly let

$$
Q_{0}:=0 \quad \text { and } \quad Q_{j+1}:=\int_{0}^{\infty} T^{*}(t)\left(D^{*} Q_{j} D-M\right) T(t) d t, \quad j \in \mathbb{N} .
$$


First we will prove the following

Claim. For all $x, y \in H$ and $j \in \mathbb{N}$ we have $\left\langle x,\left(Q_{j}^{n}-Q_{j}\right) y\right\rangle \rightarrow 0$ as $n \rightarrow \infty$. Proof of claim. For $j=0$ the claim holds trivially. Suppose now that the claim holds for value $j=k-1$. Let $x, y \in H$. Then for $j=k$,

$$
\begin{aligned}
\left|\left\langle x,\left(Q_{j}^{n}-Q_{j}\right) y\right\rangle\right|= & \mid \int_{0}^{\infty}\left\langle T_{n}(t) x,\left(D^{*} Q_{j-1}^{n} D-M\right) T_{n}(t) y\right\rangle d t \\
& -\int_{0}^{\infty}\left\langle T(t) x,\left(D^{*} Q_{j-1} D-M\right) T(t) y\right\rangle d t \mid \\
\leq & \int_{0}^{\infty}\left|\left\langle T_{n}(t) x, D^{*}\left(Q_{j-1}^{n}-Q_{j-1}\right) D T_{n}(t) y\right\rangle\right| d t \\
& +\mid\left\langle x, \int_{0}^{\infty} T_{n}^{*}(t)\left(D^{*} Q_{j-1} D-M\right) T_{n}(t) d t y\right\rangle \\
& -\left\langle x, \int_{0}^{\infty} T(t)^{*}\left(D^{*} Q_{j-1} D-M\right) T(t) d t y\right\rangle
\end{aligned}
$$

Since by the induction hypothesis $Q_{j-1}^{n} \rightarrow Q_{j-1}$ in weak sense, we have that

$$
\left\langle D T_{n}(t) x,\left(Q_{j-1}^{n}-Q_{j-1}\right) D T_{n}(t) y\right\rangle \rightarrow 0 \quad \text { as } n \rightarrow \infty
$$

for all $t \in[0, \infty)$. By dominated convergence therefore the first term proceeds zero as $n \rightarrow \infty$. The convergence of the second term is an immediate consequence of Lemma 4.2.

So the claim is proven by induction.

Now by the proof of Theorem 3.4, $Q_{j} \rightarrow Q$ and $Q_{j}^{n} \rightarrow Q^{n}$ in the norm topology of $L(H)$, uniformly in $n$. Therefore using

$\left|\left\langle x,\left(Q^{n}-Q\right) y\right\rangle\right| \leq\left|\left\langle x,\left(Q^{n}-Q_{j}^{n}\right) y\right\rangle\right|+\left|\left\langle x,\left(Q_{j}^{n}-Q_{j}\right) y\right\rangle\right|+\left|\left\langle x,\left(Q_{j}-Q\right) y\right\rangle\right|$ we obtain $\left\langle x,\left(Q^{n}-Q\right) y\right\rangle \rightarrow 0$ as $n \rightarrow \infty$.

Lemma 4.4. Let $B \in L(H)$ and let $k \in \mathbb{R}$ such that $B-k I$ is stable, i.e. we may estimate $\left\|e^{-k t} e^{B t}\right\| \leq m \mathrm{e}^{\lambda t}, t \geq 0$ for some $m \geq 1$ and $\lambda<0$.

Then for any $Q \in L(H), Q \geq 0$, and $x \in H$ we have

$$
\frac{\langle Q B x, x\rangle}{\langle Q x, x\rangle} \leq k \text {. }
$$

Proof. Let

$$
N:=(B-k I)^{*} Q+Q(B-k I) .
$$

Then by Lyapunov theory $N \leq 0$. Hence

$$
2\langle Q(B-k I) x, x\rangle=\langle N x, x\rangle \leq 0
$$


or equivalently

$$
\langle Q B x, x\rangle \leq k\langle Q x, x\rangle
$$

We are now ready to state and prove the main result of this section.

Theorem 4.5. Let $A$ be the infinitesemal generator of a strongly continuous semigroup in $L(H)$ and let $B \in L(H)$. Suppose there exists $b \in \mathbb{R}$ such that the semigroup $S(t)$ generated by $A+b B$ satisfies, for some $\mu \in \mathbb{R}$,

$$
\|S(t)\| \leq \mathrm{e}^{\mu t}, \quad t \geq 0 .
$$

Furthermore suppose that for some $\lambda \in \mathbb{R}$,

$$
2\left(\mu+\frac{1}{4} b^{2}-\lambda\right)+\|B\|^{2}<0 .
$$

Then

$$
\limsup _{t \rightarrow \infty} \frac{1}{t} \log |X(t)| \leq \lambda
$$

where $(X(t))_{t \geq 0}$ is the mild solution of

$$
d X(t)=A X(t) d t+B X(t) d W(t), \quad X(0)=x,
$$

with $W$ a one-dimensional standard Brownian motion.

Proof. Define $L:=A+b B+\left(\frac{1}{4} b^{2}-\lambda\right) I$, then $L$ is the generator of a semigroup $T(t)$ and we have

$$
\|T(t)\| \leq \mathrm{e}^{\left(\mu+\frac{1}{4} b^{2}-\lambda\right) t} .
$$

In particular, $T$ is a contraction semigroup and hence also the approximating semigroups $\left(T_{n}\right)_{n \in \mathbb{N}}$ (generated by the Yosida approximation $\left(L_{n}\right)_{n \in \mathbb{N}}$ ) are contraction semigroups. Furthermore, by (26), for any $\varepsilon>0$ there exists an $N \in \mathbb{N}$ such that for all $n \geq N$ we have

$$
\left\|T_{n}(t)\right\| \leq \mathrm{e}^{\left(\mu+\frac{1}{4} b^{2}-\lambda+\varepsilon\right) t} .
$$

Let $\varepsilon>0$, small enough such that $\mu+\frac{1}{4} b^{2}-\lambda+\frac{1}{2}\|B\|^{2}+\varepsilon<0$ and let $N$ as above. Let $\omega:=\mu+\frac{1}{4} b^{2}-\lambda+\varepsilon$. For $n \geq N$ let $Q_{n}$ be the solution given by Proposition 3.5 to

$$
L_{n}^{*} Q_{n}+Q_{n} L_{n}+B^{*} Q_{n} B=L_{n}^{*}+L_{n} .
$$

Similarly let $Q$ be the solution to

$$
L^{*} Q+Q L+B^{*} Q B=L^{*}+L .
$$

Recall that $Q_{n} \geq I, n \geq N, Q \geq I$ and

$$
\|Q\| \vee \sup _{n \geq N}\left\|Q_{n}\right\| \leq \frac{-2 \omega}{-\left(2 \omega+\|B\|^{2}\right)}
$$

Let $X_{n}$ denote the solution to

$$
d X_{n}(t)=A_{n} X_{n}(t) d t+B X_{n}(t) d W(t), \quad X_{n}(0)=x,
$$


where $A_{n}:=L_{n}-b B-\left(\frac{1}{4} b^{2}-\lambda\right) I$. Then $\left(A_{n}\right)$ is an approximation for $A$ and hence by Proposition B.3, we have almost surely that, for a subsequence $\left(n_{k}\right) \subset \mathbb{N}$,

$$
\sup _{t \in[0, T]}\left|X_{n_{k}}(t)-X(t)\right| \rightarrow 0 \quad \text { for all } T>0 .
$$

Since $L_{n}$ is bounded, we have that, since

$$
\left(A_{n}+b B\right)^{*} Q_{n}+Q_{n}\left(A_{n}+b B\right)+B^{*} Q_{n} B+\left(\frac{1}{2} b^{2}-2 \lambda\right) Q_{n} \leq 0, \quad n \in \mathbb{N},
$$

and by the proofs of Lemma 3.2 and Proposition 3.3 that

$$
\log \left\langle Q_{n} X_{n}(t), X_{n}(t)\right\rangle \leq \log \left\langle Q_{n} x, x\right\rangle+2 \lambda t+2 \int_{0}^{t} \frac{\left\langle Q_{n} B X_{n}(s), X_{n}(s)\right\rangle}{\left\langle Q_{n} X_{n}(s), X_{n}(s)\right\rangle} d W(s) .
$$

Here we used that $L_{n}+L_{n}^{*} \leq 0$ by dissipativeness of $L_{n}$.

In the stochastic integral we have by Lemma 4.3 , the almost sure convergence of $X_{n}(s)$ on $[0, t]$ and the uniform boundedness of $\left\|Q_{n}\right\|, n \in \mathbb{N}$, that the integrand converges almost surely. By Lemma 4.4 we may apply dominated convergence to have convergence in $L^{2}(\Omega)$ of the stochastic integral

$$
\int_{0}^{t} \frac{\left\langle Q_{n} B X_{n}(s), X_{n}(s)\right\rangle}{\left\langle Q_{n} X_{n}(s), X_{n}(s)\right\rangle} d W(s) \rightarrow \int_{0}^{t} \frac{\langle Q B X(s), X(s)\rangle}{\langle Q X(s), X(s)\rangle} d W(s) .
$$

We therefore also have this convergence with probability one for a further subsequence $\left(n_{k_{l}}\right) \subset \mathbb{N}$.

For this subsequence the left-hand side of (14) converges almost surely to $\log \langle Q X(t), X(t)\rangle$, and by the uniform estimate on $\left\|Q_{n}\right\|$, the term $\log \left\langle Q_{n} x, x\right\rangle$ is bounded by a constant, say $M>0$. Hence we have that, with probability one,

$$
\log \langle Q X(t), X(t)\rangle \leq M+2 \lambda t+2 \int_{0}^{t} \frac{\langle Q B X(s), X(s)\rangle}{\langle Q X(s), X(s)\rangle} d W(s), \quad \text { for all } t \geq 0 .
$$

Dividing by $t$ and by letting $t \rightarrow \infty$, using the law of large numbers for martingales (Theorem A.1) we obtain

$$
\limsup _{t \rightarrow \infty} \frac{1}{t} \log \langle Q X(t), X(t)\rangle \leq 2 \lambda \quad \text { almost surely. }
$$

Using the fact that $Q \geq I$, we now have

$$
\limsup _{t \rightarrow \infty} \frac{1}{t} \log |X(t)| \leq \lambda \quad \text { almost surely. }
$$




\section{Example: Stochastic Heat Equation}

As a simple illustrative example, let $H=L^{2}(\mathcal{O})$ where $\mathcal{O}$ is some Lipschitz domain in $\mathbb{R}^{n}$. Consider the stochastic partial differential equation

$$
\begin{cases}d u(t, \xi)=[\Delta u(t, \xi)+\nu u(t, \xi)] d t+\sigma u(t, \xi) d W(t), & \xi \in \mathcal{O}, t \geq 0 \\ u(0, \xi)=v(\xi), & \xi \in \mathcal{O}, \\ \frac{\partial u(t, \xi)}{\partial n}=0, & \xi \in \partial \mathcal{O}, t \geq 0\end{cases}
$$

where $\Delta$ is the Laplace operator on $\mathcal{O}, \frac{\partial u}{\partial n}$ the partial derivative in the direction of the gradient, $\nu, \sigma \in \mathbb{R}$, and $v \in L^{2}(\mathcal{O})$ is an initial condition.

Define the generator $A$ of the corresponding strongly continuous semigroup $(T(t))_{t \geq 0}$ on $L^{2}(\mathcal{O})$ by $A=\Delta+\nu I$. Let $B=\sigma I$. Let $b \in \mathbb{R}$ and note that the semigroup $(S(t))$ generated by $A+b B=\Delta+(\nu+\sigma) I$ satisfies $\| S(t)) \| \leq \mathrm{e}^{(\nu+b \sigma) t}$. By Theorem 4.5, if

$$
\lambda>\nu+b \sigma+\frac{1}{4} b^{2}+\frac{1}{2} \sigma^{2},
$$

then the solution of (15) satisfies

$$
\limsup _{t \rightarrow \infty} \frac{1}{t} \log |u(t, \cdot)|_{L^{2}(\mathcal{O})} \leq \lambda .
$$

The optimal lower estimate for $\lambda$ is obtained by choosing $b=-2 \sigma$. This results in the conclusion that for $\lambda>\nu-\frac{1}{2} \sigma^{2}$ we have that (16) holds. Note the stabilizing effect of the noise.

This result is in agreement with the estimate given by (4) on commuting operators. It could well have been derived with the results of [11] or [23], and is included here mainly to provide an easy example and to show that we are able with our method to obtain this sharpest obtainable bound.

\section{Example: Stochastic Delay Differential Equation}

Consider the stochastic differential equation with delay

$$
d Y(t)=a Y(t)+c Y(t-\tau) d t+\sigma Y(t) d W(t), \quad t \geq 0, \quad Y(0)=y .
$$

with $a, c \in \mathbb{R}$ and $\tau, \sigma>0$.

Suppose first $c=0$. Then the solution to the stochastic differential equation is given by

$$
Y(t)=\exp \left(\left(a-\frac{1}{2} \sigma^{2}\right) t+\sigma W(t)\right) y, \quad t \geq 0,
$$

and the solution is pathwise asymptotically stable if $a<\frac{1}{2} \sigma^{2}$.

We may now ask ourselves the question: for which $c \in \mathbb{R}$ do we still have stability?

Theorem 6.1. Suppose $a<\frac{1}{2} \sigma^{2}$ and

$$
|c|<\mathrm{e}^{-\frac{3}{2} \sigma^{2} \tau}\left(\frac{1}{2} \sigma^{2}-a\right) .
$$

Then the solution to (17) is pathwise exponentially stable. 
Proof. For convenience we recast the SDDE (17) in an SDDE with delay time equal to one. By letting $\widetilde{Y}(t):=Y(\tau t)$ and $\widetilde{W}(t)=W(t \tau) / \sqrt{\tau}$, the problem may be rewritten as

$$
d \widetilde{Y}(t)=(\tilde{a} \widetilde{Y}(t)+\tilde{c} \widetilde{Y}(t-1)) d t+\tilde{\sigma} \widetilde{Y}(t) d \widetilde{W}(t),
$$

with $\tilde{a}=\tau a, \tilde{c}=\tau c$ and $\tilde{\sigma}=\sqrt{\tau} \sigma$. For the remainder of the proof we will omit the tildes.

As in Section $\mathrm{C}$, let $A$ be the generator of the delay semigroup in $\mathbb{R} \times$ $L^{2}([-1,0])$, with

$$
A:=\left[\begin{array}{ll}
a & \Phi \\
0 & \frac{d}{d \sigma}
\end{array}\right],
$$

where $\Phi u:=c u(-1)$ for $u \in W^{1,2}([-1,0])$. Furthermore let $B \in L(\mathbb{R} ; \mathbb{R} \times$ $\left.L^{2}([-1,0])\right)$ be given by

$$
B:=\left[\begin{array}{ll}
\sigma & 0 \\
0 & 0
\end{array}\right]
$$

By Theorem C.1, we have that if, for some $b \in \mathbb{R}$,

$$
(a+b \sigma-\mu)^{2}>c^{2} \mathrm{e}^{-2 \mu}
$$

and

$$
\mu>a+b \sigma
$$

then $A+b B-\mu I$ generates a dissipative semigroup on a renormed space $\mathbb{R} \times L^{2}([-1,0], \tau)$ (with $L^{2}([-1,0], \tau)$ the Hilbert space consisting of square integrable functions on $[0,1]$ with inner product

$$
\langle f, g\rangle_{\tau}=\int_{-1}^{0} f(s) g(s) \tau(s) d s
$$

for some suitable weight function $\tau \in L^{\infty}([-1,0])$.

By Theorem 4.5, we have that (17) has a pathwise exponentially stable solution if

$$
\sigma^{2}+\frac{1}{2} b^{2}+2 \mu<0 .
$$

We may reformulate (19) as

$$
c^{2} \leq(a+b \sigma-\mu)^{2} \mathrm{e}^{2 \mu},
$$

where $b$ and $\mu$ should satisfy (20) and (21). It may be verified that $b=-2 \sigma$ and $\mu=-3 / 2 \sigma^{2}-\varepsilon$, with $\varepsilon>0$ sufficiently small, satisfy these conditions, using that $a<\frac{1}{2} \sigma^{2}$. Now recall the substitutions $a \rightarrow \tau a, c \rightarrow \tau c$ and $\sigma \rightarrow \sqrt{\tau} \sigma$ and let $\varepsilon \downarrow 0$ to obtain the estimate (18).

We may rephrase the estimate (18) as a condition on the delay time: assume $|c|<\frac{1}{2} \sigma^{2}-a$ and

$$
\tau<\frac{2}{3 \sigma^{2}} \ln \left(\frac{\frac{1}{2} \sigma^{2}-a}{|c|}\right) .
$$

Then the solution of (17) is almost surely exponentially stable. 
Remark 6.2. From the proof of Theorem 6.1, we see that the best estimate for $|c|$ in order for the system to remain asymptotically stable (with $\tau=1$, for convenience) is obtained by solving the nonlinear optimization problem

$$
\begin{aligned}
\max & (a+b \sigma-\mu)^{2} \mathrm{e}^{2 \mu} \\
\text { subject to } & a+b \sigma-\mu<0 \\
\text { and } & \sigma^{2}+\frac{1}{2} b^{2}+2 \mu<0 .
\end{aligned}
$$

over $b$ and $\mu$. The condition $a<\frac{1}{2} \sigma^{2}$ is required for the set of feasible $(b, \sigma)$ to be non-empty. The above problem may be solved by applying the KarushKuhn-Tucker conditions, obtaining $\mu=-\frac{1}{4} b^{2}-\frac{1}{2} \sigma^{2}$ and for $b$ the third-degree equation

$$
\frac{1}{4} b^{3}+b^{2} \sigma+b\left(a+\frac{1}{2} \sigma^{2}-1\right)-2 \sigma=0 .
$$

By solving this equation we obtain an estimate which is sharper than (18), but less readable.

Remark 6.3. Note that, for $\sigma=0$, we obtain from (18) the condition $|c|<-a$, which is the same estimate as that in Corollary C.2. Furthermore we have

$$
\left.\frac{d}{d\left(\sigma^{2}\right)} \mathrm{e}^{-3 / 2 \sigma^{2}}\left(\frac{1}{2} \sigma^{2}-a\right)\right|_{\sigma^{2}=0}=\frac{1}{2}(3 a+1),
$$

from which we may conclude (see (18)) that adding noise has a stabilizing effect for $a>-\frac{1}{3}$.

Example 6.4. (Population growth under random migration) Consider a population $(x(t))_{t \geq 0}$ evolving with constant birth rate $\beta>0$ and constant death rate $\alpha>0$. Let $r=1$ indicate the development period of an individual. Suppose there is migration with random rate $\sigma$ which may depend on the size of the population. This leads to the stochastic differential equation with delay

$$
d x(t)=[-\alpha x(t)+\beta x(t-1)] d t+\sigma x(t) d W(t), \quad t>0 .
$$

so in (17) we have $a=-\alpha$ and $c=\beta$. Then Theorem 6.1 tells us that for $\beta<\mathrm{e}^{-3 / 2 \sigma^{2}}\left(\frac{1}{2} \sigma^{2}+\alpha\right)$ the population will eventually be extinguished with probability one.

If, for example $\alpha=0.1$, then the graph in Fig. 1 shows upper bounds on values of $\beta$ for which we know to have pathwise stability.

Numerical experiments suggest that for $\sigma$ not too large the theoretical bounds are quite accurate (see Fig. 2).

\subsection{Comparison and Discussion}

Note that, under the conditions of Theorem 6.1, we do not necessarily have moment stability. Almost all papers on almost sure or pathwise stability of stochastic delay differential equations (e.g. [26, 28-30]) first establish moment stability and then state sufficient conditions such that moment stability implies pathwise stability. In particular it is usually required that $a+|c|<$ $-\frac{1}{2} \sigma^{2}$, which should be compared with our 'basic assumption' $a+|c|<\frac{1}{2} \sigma^{2}$. This shows that our result on pathwise stability is much stronger than those in the mentioned papers. 


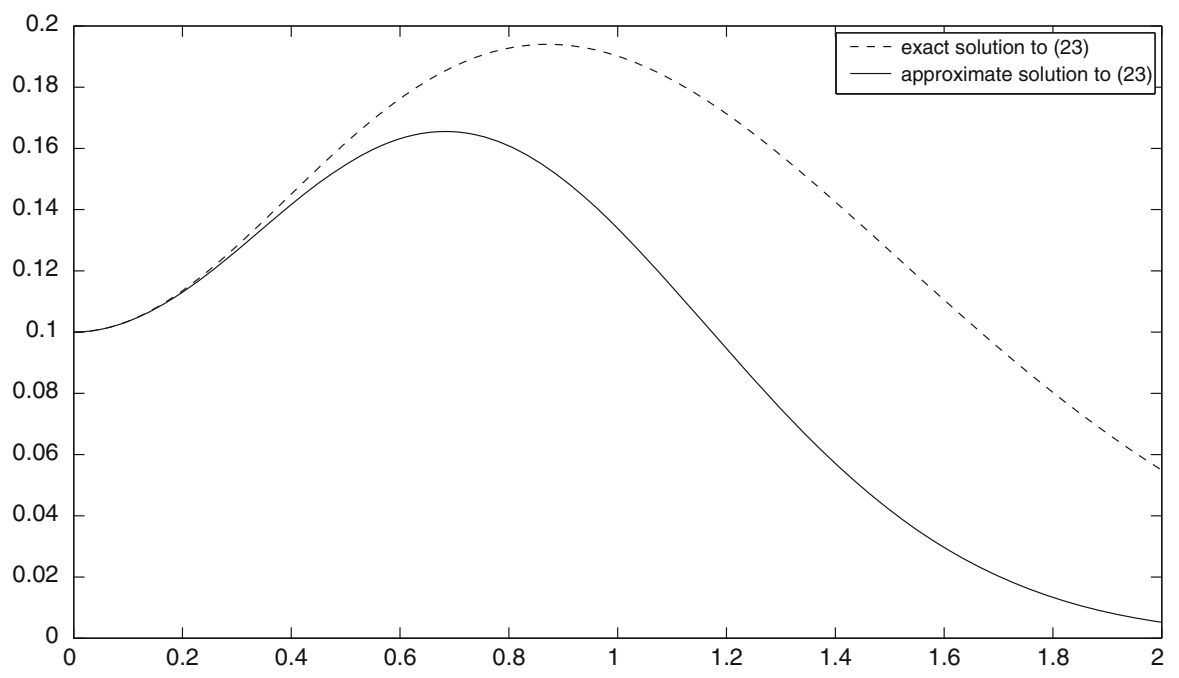

FiguRE 1. Bound on the birth rate for which the population is eventually extinguished, as function of $\sigma$. Here $\alpha=0.1$. The solid graph represents the approximation $\exp \left(-3 / 2 \sigma^{2}\right)\left(\frac{1}{2} \sigma^{2}+\alpha\right)$ provided by Theorem 6.1. The dashed graph represent the exact solution to the optimization problem (23), which gives a more relaxed requirement on $\beta$

An exception is the beautiful result of [1] by Appleby and Mao, which also applies also to nonlinear SDDEs. In the context of our paper, they obtain the following upper bound on $c$ :

$$
|c|<-a+\frac{1}{4} \sigma^{2} \mathrm{e}^{-\sigma^{2} \tau} / \Phi(\sigma \sqrt{\tau}),
$$

where $\Phi$ is the CDF of the standard normal distribution. This result may be compared to our bound (18). For some values of parameters $a, \sigma$ and $\tau$ our results are sharper, in particular for larger values of $a$ and $\sigma$, see Fig. 3.

In the derivation, completely different methods were used in order to obtain these results. The result of [1] is derived explicitly for stochastic delay differential equations. In fact, their result naturally leads to the question: can we obtain a similar result on pathwise stability by taking a more operator theoretic point of view, in the style of Da Prato and Zabczyk [16], to which this paper provides a partial answer. In [11] such operator theoretic results are obtained for stochastic evolutions, but these require a special form of the noise and are therefore not applicable to stochastic delay differential equations.

The general result of our analysis, Theorem 4.5, is applicable to general stochastic evolutions.

\section{Notes and Remarks}

The part on stability of degenerate evolutions with bounded generator (applied to finite dimensional SDEs) will be published separately [8]. 


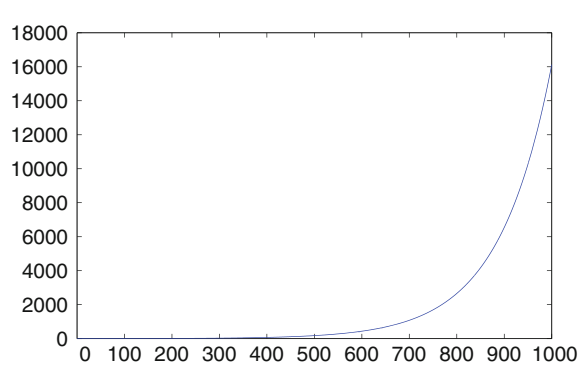

(a) No migration, $\sigma=0$

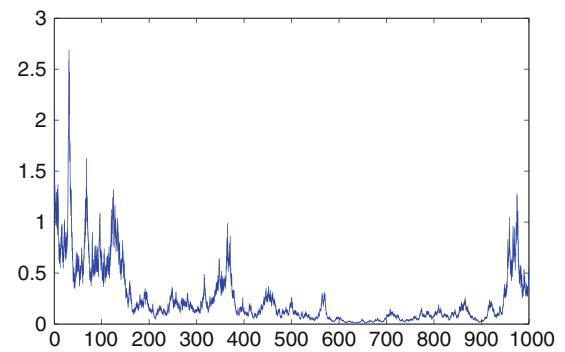

(c) Random migration at rate $\sigma=0.2$

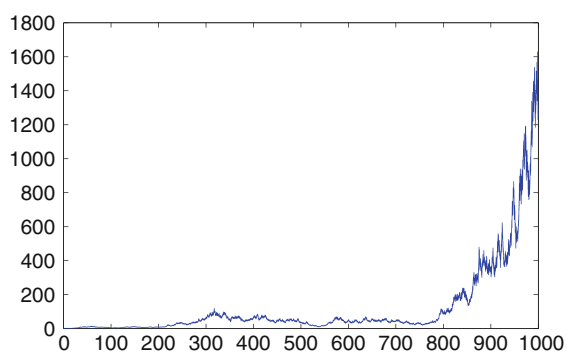

(b) Random migration at rate $\sigma=0.1$

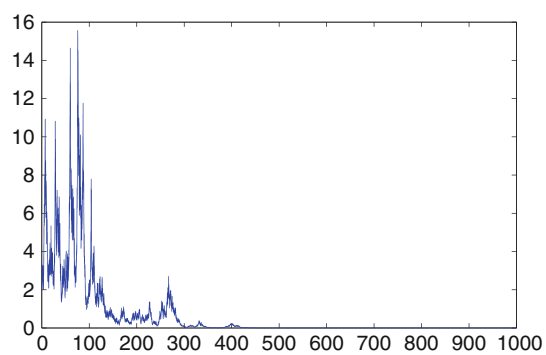

(d) Random migration at rate $\sigma=0.3$

Figure 2. The evolution of the size of a population in time with birth rate $\beta=0.11$ and death rate $\alpha=0.1$. Without migration or for a small rate of random migration the population increases steadily in size, whereas for a larger rate of migration the population is eventually extinguished

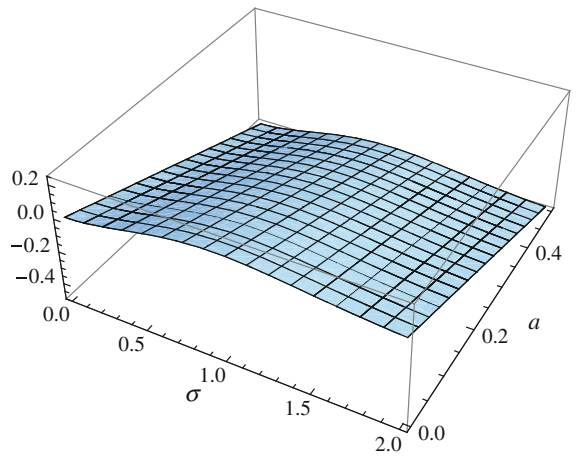

(a) Result of [1]

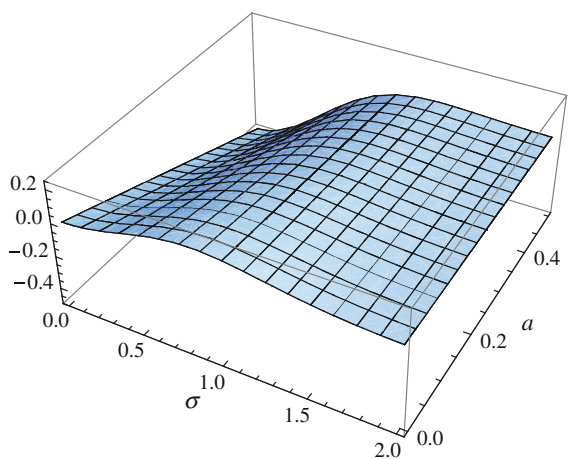

(b) Our result

Figure 3. Comparison of bound on $|c|$, depending on $\sigma$ and $a$. Here the delay time $\tau=1$

For more results on finite dimensional stochastic Lyapunov exponents, see the overview paper [4], the book by Khasminskii [22], and e.g. [2,3,24,31] and [33].

Results on pathwise stability with general decay rate (i.e. not necessarily exponential decay) of stochastic evolutions are given in [9]. In [10] results 
on stabilization by noise of some partial differential equations may be found. In [12] results on stabilization by noise for stochastic reaction-diffusion equations are used to establish existence and uniqueness of invariant measure.

In [25] a result on pathwise stability of finite dimensional stochastic differential equations with jumps is established, using the existence of an invariant measure for the projection of the solution on the unit sphere.

An insightful discussion on Itô noise versus Stratonovich noise may be found in [11], together with results on pathwise stability of linear stochastic partial differential equations with linear multiplicative noise.

\section{Acknowledgments}

The author wishes to express his thanks to Onno van Gaans and Sjoerd Verduyn Lunel (Mathematical Institute, Leiden) and also the anonymous reviewer for their insightful remarks which added substantially to the quality of this paper.

Open Access. This article is distributed under the terms of the Creative Commons Attribution Noncommercial License which permits any noncommercial use, distribution, and reproduction in any medium, provided the original author(s) and source are credited.

\section{Appendix A. Law of Large Numbers for Martingales}

In this paper we make use of the law of large numbers for martingales. This is not a new result; a more general formulation can be found for example in [29], Theorem 3.4 but without proof. A proof for the formulation below may be found in [6] or [8].

Theorem A.1. (Law of large numbers for martingales) Let $(M(t))_{t \geq 0}$ be $a$ continuous local martingale in $\mathbb{R}$ with $M(0)=0$. If

$$
\limsup _{t \rightarrow \infty} \frac{[M](t)}{t}<\infty, \quad \text { a.s. }
$$

then

$$
\lim _{t \rightarrow \infty} \frac{M(t)}{t}=0, \quad \text { a.s. }
$$

\section{Appendix B. Approximation of Solutions of Stochastic Differential Equations}

To be able to extend results from the case of uniformly continuous semigroups to the more general case of strongly continuous semigroups, we will need some results on approximation of solutions of SDEs.

The notion of Yosida approximations is well known in semigroup theory, but too restrictive for our purposes. We wish to allow certain perturbations of the Yosida approximation by bounded operators. This leads to our slightly more general notion of approximations which we define now. 
Definition B.1. Let $X$ be a Banach space. Let $A$ be the infinitesemal generator of a strongly continuous semigroup $(S(t))_{t \geq 0}$ in $L(X)$. Let $\left(T_{n}(t)\right)_{t \geq 0, n \in \mathbb{N}}$ be a sequence of strongly continuous semigroups in $L(X)$ with generators $\left(A_{n}\right)_{n \in \mathbb{N}}$.

Then $\left(A_{n}\right)$ is called an approximation of $A$ if

(i) there exists $M>0$ and $\omega \in \mathbb{R}$ such that

$$
\|T(t)\| \vee \sup _{n \in \mathbb{N}}\left\|T_{n}(t)\right\| \leq M \mathrm{e}^{\omega t} \quad \text { for all } t \geq 0
$$

and

(ii) we have that $T_{n}(t) x \rightarrow T(t) x$ as $n \rightarrow \infty$ for all $x \in X$, uniformly in $t$ on compact sets.

An approximation $\left(A_{n}\right)$ of $A$ is called a bounded approximation if $A_{n} \in$ $L(X)$ for all $n \in \mathbb{N}$.

We will not distinguish between an approximation $\left(A_{n}\right)$ of $A$ or an approximation $\left(T_{n}\right)$ of the corresponding semigroup $T$.

Equivalent conditions for $\left(A_{n}\right)$ to be an approximation of $A$ are given by the Trotter-Kato theorem, see [18], Theorem III.4.8. A sufficient condition for a sequence $\left(A_{n}\right)$ to be an approximation of $A$ is that (25) holds, and that $A_{n} x \rightarrow A x$ for all $x \in D$, where $D$ is a core for $A$.

\section{B.1. Yosida Approximation}

An important example of bounded approximations is the Yosida approximation which we will discuss here.

Let $X$ be a Banach space. In this example, $A: \mathfrak{D}(A) \rightarrow X$ is the infinitesemal generator of a strongly continuous semigroup $(S(t))_{t \geq 0}$ on $X$ satisfying

$$
\|S(t)\| \leq M \mathrm{e}^{\omega t}, \quad t \geq 0
$$

where $M \geq 1$ and $\omega \in \mathbb{R}$.

Recall the notions of the resolvent set of $A$,

$$
\rho(A):=\{\lambda \in \mathbb{C}: \lambda-A \text { has a bounded inverse }\},
$$

and the resolvent of $A$,

$$
R(\lambda, A):=(\lambda-A)^{-1}, \quad \lambda \in \rho(A) .
$$

Define the Yosida approximation of $A$ by

$$
A_{n}:=A J_{n}=n A R(n, A)=n^{2} R(n, A)-n I, \quad n \in \mathbb{N} \cap \rho(A),
$$

where $J_{n}:=n R(n, A)$. These $\left(A_{n}\right)_{n \in \mathbb{N}}$ are bounded operators and therefore generate uniformly continuous semigroups which we denote by $\left(S_{n}(t)\right)_{t \geq 0}$, $n \in \mathbb{N}$. Furthermore $A_{n} x \rightarrow A x$ for all $x \in \mathfrak{D}(A)$.

By [15], Theorem A.2,

$$
\left\|S_{n}(t)\right\| \leq M \mathrm{e}^{\frac{\omega n t}{n-\omega}}, \quad t \geq 0 .
$$

Note that

$$
\frac{\omega n}{n-\omega} \rightarrow \omega
$$


In particular, for all $\widetilde{\omega}>\omega$ there exists an $N \in \mathbb{N}$ such that for and all $n>N$, we have

$$
\left\|S_{n}(t)\right\| \leq M \mathrm{e}^{\widetilde{\omega} t}, \quad t \geq 0 .
$$

If we combine the Trotter-Kato approximation theorem [18, Theorem III.4.8] with (26), we obtain the following lemma:

Lemma B.2. $S_{n}(t) x \rightarrow S(t) x$ for all $x \in X$, uniformly for $t \in[0, T]$ with $T>0$.

\section{B.2. Convergence of Stochastic Evolutions}

Proposition B.3. Suppose $S$ is a strongly continuous semigroup with infinitesemal generator $A, W$ is a standard Brownian motion in $\mathbb{R}^{m}, p>2$ and $X_{0} \in L^{p}\left(\Omega, \mathcal{F}_{0} ; H\right)$. Suppose $\left(A_{n}\right)_{n \in \mathbb{N}}$ are approximations of $A$. Suppose $F$ : $H \rightarrow H$ and $G: H \rightarrow L\left(\mathbb{R}^{m} ; H\right)$ are globally Lipschitz. Let $X$ be the unique mild solution to

$$
d X(t)=(A X+F(X)) d t+G(X) d W(t), \quad X(0)=X_{0},
$$

and $X_{n}$ the unique mild solution to

$$
d X(t)=\left(A_{n} X_{n}+F\left(X_{n}\right)\right) d t+G\left(X_{n}\right) d W(t), \quad X(0)=X_{0} .
$$

Then for all $T>0$,

$$
\mathbb{E} \sup _{t \in[0, T]}\left|X(t)-X_{n}(t)\right|^{p} \rightarrow 0 \quad \text { as } \quad n \rightarrow \infty .
$$

Moreover, there exists a sequence $\left(n_{k}\right)_{k \in \mathbb{N}}$ in $\mathbb{N}$ such that

$$
\lim _{k \rightarrow \infty} \sup _{t \in[0, T]}\left|X_{n_{k}}(t)-X(t)\right| \quad \text { for all } T>0, \quad \text { almost surely. }
$$

Proof. Let $\varepsilon>0$.

$$
\begin{aligned}
& \mathbb{E}\left[\sup _{t \in[0, T]}\left|X(t)-X_{n}(t)\right|^{p}\right] \\
& \leq 3^{p-1} \mathbb{E}\left[\sup _{t \in[0, T]}\left|S(t) X_{0}-S_{n}(t) X_{0}\right|^{p}\right] \\
& +3^{p-1} \mathbb{E}\left[\left.\sup _{t \in[0, T]}\left|\int_{0}^{t} S(t-s) F(X(s))-S_{n}(t-s) F\left(X_{n}(s)\right) d s\right|\right|^{p}\right] \\
& +3^{p-1} \mathbb{E}\left[\sup _{t \in[0, T]}\left|\int_{0}^{t} S(t-s) G(X(s))-S_{n}(t-s) G\left(X_{n}(s), s\right) d W(s)\right|^{p}\right] .
\end{aligned}
$$

First term. For the first term we have that

$$
\lim _{n \rightarrow \infty} \sup _{t \in[0, T]}\left|S(t) X_{0}-S_{n}(t) X_{0}\right|^{2} \rightarrow 0, \quad \text { almost surely. }
$$


Since $(S(t))_{t \in[0, T]}$ and $\left(S_{n}(t)\right)_{t \in[0, T]}$ are uniformly bounded in operator norm, by dominated convergence,

$$
3^{p-1} \mathbb{E}\left[\sup _{t \in[0, T]}\left|S(t) X_{0}-S_{n}(t) X_{0}\right|^{2}\right]<\varepsilon
$$

for $n$ large enough.

Second term. Note that

$$
\begin{aligned}
& \sup _{s \in[0, t]}\left|\int_{0}^{s} S(s-r) F(X(r))-S_{n}(s-r) F\left(X_{n}(r)\right) d r\right|^{p} \\
& \leq \sup _{s \in[0, t]} s^{p-1} \int_{0}^{s}\left|S(s-r) F(X(r))-S_{n}(s-r) F\left(X_{n}(r)\right)\right|^{p} d r \\
& \leq \sup _{s \in[0, t]}(2 s)^{p-1} \int_{0}^{s}\left|S_{n}(s-r)\left(F(X(r))-F\left(X_{n}(r)\right)\right)\right|^{p} d r \\
& \quad+\sup _{s \in[0, t]}(2 s)^{p-1} \int_{0}^{s}\left|S_{n}(s-r) F(X(r))-S(s-r) F(X(r))\right|^{p} d r .
\end{aligned}
$$

with

$$
\begin{aligned}
& \sup _{s \in[0, t]} \int_{0}^{s}\left|S_{n}(s-r)\left(F(X(r))-F\left(X_{n}(r)\right)\right)\right|^{p} d r \\
& \leq k_{1} \int_{0}^{t} \sup _{r \in[0, s]}\left|X(r)-X_{n}(r)\right|^{p} d s
\end{aligned}
$$

where the constant $k_{1}>0$ may be chosen such that the inequality holds for all $t \in[0, T]$. Furthermore by dominated convergence, for $n$ large enough,

$$
\mathbb{E} \sup _{s \in[0, t]}(2 s)^{p-1} \int_{0}^{s}\left|S_{n}(s-r) F(X(r))-S(s-r) F(X(r))\right|^{p} d r<\varepsilon .
$$

Third term. For the third term, estimate the stochastic convolution twice (using [15, Proposition 7.3]), for $n$ large enough,

$$
\begin{aligned}
& \mathbb{E} \sup _{s \in[0, t]}\left|\int_{0}^{s} S(s-r) G(X(r))-S_{n}(s-r) G\left(X_{n}(r)\right) d W(r)\right|^{p} \\
& \leq 2^{p-1} \mathbb{E} \sup _{s \in[0, t]}\left|\int_{0}^{s} S(s-r) G(X(r))-S_{n}(s-r) G(X(r)) d W(s)\right|^{p}
\end{aligned}
$$




$$
\begin{aligned}
& +2^{p-1} \mathbb{E} \sup _{s \in[0, t]}\left|\int_{0}^{s} S_{n}(s-r)\left(G(X(r))-G\left(X_{n}(r)\right)\right) d W(s)\right|^{p} \\
& \leq \varepsilon+k_{2} \mathbb{E} \int_{0}^{t} \sup _{r \in[0, s]}\left|X(r)-X_{n}(r)\right|^{p} d t,
\end{aligned}
$$

where the constant $k_{2}>0$ may be chosen in such a way that the inequality holds for all $t \in[0, T]$.

Combining all the terms, for $t \in[0, T]$ and for $n$ large enough,

$$
\begin{aligned}
& \mathbb{E} \sup _{s \in[0, t]}\left|X(s)-X_{n}(s)\right|^{p} \\
& \quad \leq 3 \varepsilon+\left(k_{1}+k_{2}\right) \int_{0}^{t} \mathbb{E} \sup _{r \in[0, s]}\left|X(r)-X_{n}(r)\right|^{p} d s .
\end{aligned}
$$

By Gronwall's lemma therefore, for $t \in[0, T]$,

$$
\mathbb{E} \sup _{s \in[0, t]}\left|X(s)-X_{n}(s)\right|^{p} \leq 3 \varepsilon \exp \left(\left(k_{1}+k_{2}\right) t\right),
$$

and we may let $\varepsilon \downarrow 0$ to obtain the first claim. It follows that for every $T>0$ there exists a subsequence $(n(k))_{k \in \mathbb{N}}$ such that

$$
\lim _{k \rightarrow \infty} \sup _{t \in[0, T]}\left|X(t)-X_{n(k)}(t)\right|=0, \quad \text { a.s. }
$$

Now define $\Omega_{1} \subset \Omega, \mathbb{P}\left(\Omega_{1}\right)=1$ and a subsequence $\left(n_{1}(k)\right)_{k \in \mathbb{N}}$ such that

$$
\lim _{k \rightarrow \infty} \sup _{t \in[0,1]}\left|X_{n_{1}(k)}(t)-X(t)\right|=0 \quad \text { on } \Omega_{1} .
$$

Define recursively, for $m \in \mathbb{N}, m \geq 2$, sets $\Omega_{m} \subset \Omega, \mathbb{P}\left(\Omega_{m}\right)=1$, and further subsequences $\left(n_{m}(k)\right)_{k \in \mathbb{N}}$ of $\left(n_{m-1}(k)\right)_{k \in \mathbb{N}}$ such that

$$
\lim _{k \rightarrow \infty} \sup _{t \in[0, m]}\left|X_{n_{m}(k)}(t)-X(t)\right|=0 \quad \text { on } \Omega_{m} .
$$

Let $\tilde{\Omega}:=\cap_{m \in \mathbb{N}} \Omega_{m}$ (so $\left.\mathbb{P}(\tilde{\Omega})=1\right)$, and consider the subsequence $\left(n_{k}(k)\right)_{k \in \mathbb{N}}$. Let $\omega \in \tilde{\Omega}, \varepsilon>0$ and $M \in \mathbb{N}$. Take $K>0$ such that

$$
\sup _{t \in[0, M]}\left|X_{n_{M}}(k)(t)-X(t)\right|(\omega)<\varepsilon \quad \text { for all } k \geq K .
$$

Then for $k \geq K \vee M, n_{k}(k) \geq n_{M}(k)$, and $n_{k}(k) \in\left(n_{M}(l)\right)_{l \in \mathbb{N}}$, and therefore

$$
\sup _{t \in[0, M]}\left|X_{n_{k}}(k)(t)-X(t)\right|(\omega)<\varepsilon .
$$

This proves the second claim: There exists a set $\tilde{\Omega}$ with $\mathbb{P}(\tilde{\Omega})=1$ and a subsequence $\left(n_{k}(k)\right)_{k \in \mathbb{N}}$ of $\mathbb{N}$ such that on $\tilde{\Omega}$, we have that for any $M \in \mathbb{N}$ (and hence any $T>0$ ),

$$
\lim _{k \rightarrow \infty} \sup _{t \in[0, M]}\left|X_{n_{k}(k)}(t)-X(t)\right|=0 .
$$




\section{Appendix C. Stochastic Delay Differential Equations}

In this section we provide the preliminaries for stochastic delay differential equations.

Consider first a deterministic linear delay differential equation in $\mathbb{R}^{n}$ of the following form

$$
\left\{\begin{array}{l}
\dot{x}(t)=A_{0} x(t)+\sum_{i=1}^{k} A_{i} x\left(t-\theta_{i}\right) \\
x(0)=c \\
x(t)=f(t), \quad-1 \leq t<0
\end{array}\right.
$$

with $A_{i} \in L\left(\mathbb{R}^{n}\right), i=0, \ldots, k$, and $\theta_{i} \in(0,1], i=1, \ldots, k, c \in \mathbb{R}^{n}$ and $f \in L^{2}\left([-1,0] ; \mathbb{R}^{n}\right)$. See $[5,17]$ and [13], Section 2.4. This equation has a unique solution $(x(t))_{t \geq-1}$. Denote the segment process by $x_{t}$, where $x_{t} \in$ $L^{2}\left([-1,0] ; \mathbb{R}^{n}\right)$ is defined by $x_{t}(s)=x(t+s), t \geq 0, s \in[-1,0]$. The segment process keeps track of the 'history' of the delay equation.

As discussed there, we may define a strongly continuous semigroup $(T(t))$, called the delay semigroup acting on the state space $\mathcal{E}^{2}:=\mathbb{R}^{n} \times$ $L^{2}\left([-1,0] ; \mathbb{R}^{n}\right)$ with infinitesemal generator $A$ given by

$$
A\left(\begin{array}{l}
c \\
f
\end{array}\right)=\left(\begin{array}{c}
A_{0} c+\sum_{i=1}^{k} A_{i} f\left(-\theta_{i}\right) \\
\frac{d}{d s} f
\end{array}\right)
$$

with domain

$$
\mathcal{D}(A)=\left\{\left(\begin{array}{l}
c \\
f
\end{array}\right) \in \mathcal{E}^{2}: f \in W^{1,2}\left([-1,0] ; \mathbb{R}^{n}\right), f(0)=c\right\} .
$$

Applying this semigroup to an initial condition $\left(\begin{array}{l}c \\ f\end{array}\right) \in \mathcal{E}^{2}$, corresponds to solving the delay differential equation (27):

$$
\left(\begin{array}{c}
x(t) \\
x_{t}
\end{array}\right)=T(t)\left(\begin{array}{c}
c \\
f
\end{array}\right) \text {. }
$$

We may now add nonlinear disturbances and noise. Consider the stochastic differential equation with delay,

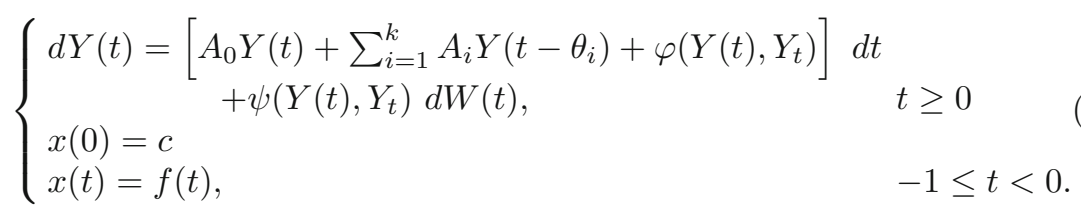

with $(W(t))$ an $m$-dimensional standard Brownian motion, $\varphi: \mathcal{E}^{2} \rightarrow \mathbb{R}^{n}$ Lipschitz and $\psi: \mathcal{E}^{2} \rightarrow L\left(\mathbb{R}^{m} ; \mathbb{R}^{n}\right)$ Lipschitz.

Define $F: \mathcal{E}^{2} \rightarrow \mathcal{E}^{2}$ and $G: \mathcal{E}^{2} \rightarrow L\left(\mathbb{R}^{m} ; \mathcal{E}^{2}\right)$ by

$$
F\left(\left(\begin{array}{l}
c \\
f
\end{array}\right)\right):=\left(\begin{array}{c}
\varphi(c, f) \\
0
\end{array}\right), \quad G\left(\left(\begin{array}{l}
c \\
f
\end{array}\right)\right):=\left(\begin{array}{c}
\psi(c, f) \\
0
\end{array}\right) .
$$


Then $F$ and $G$ are Lipschitz mappings. We may write (28) as a stochastic evolution in $\mathcal{E}^{2}$ as

$$
\left\{\begin{array}{l}
d X(t)=[A X(t)+F(X(t))] d t+G(X(t)) d W(t), \quad t \geq 0 \\
X(0)=\left(\begin{array}{l}
c \\
f
\end{array}\right) .
\end{array}\right.
$$

This equation should always be interpreted in mild form, i.e.

$$
X(t)=T(t)\left(\begin{array}{l}
c \\
f
\end{array}\right)+\int_{0}^{t} T(t-s) F(X(s)) d s+\int_{0}^{t} T(t-s) G(X(s)) d W(s) .
$$

Then $X(t)=\left(\begin{array}{c}Y(t) \\ Y_{t}\end{array}\right)$ provides the unique solution of (28). See also [16, Chapter 10] and [6, Chapter 3].

In the example in this paper we are only concerned with the one-dimensional case, $n=m=1$, where the linear operators $\left(A_{i}\right)$ become real constants $\left(a_{i}\right)$, and where $\varphi=0$ and $\psi(c, f)=\sigma c$, with $\sigma \in \mathbb{R}$, corresponding to the linear equation

$$
d Y(t)=\left[a_{0} Y(t)+\sum_{i=1}^{k} a_{i} Y\left(t-\theta_{i}\right)\right] d t+\sigma Y(t) d W(t)
$$

with $W$ a one-dimensional standard Brownian motion.

We will now quote a method to transform the delay semigroup into a generalized contraction semigroup by changing the inner product on $\mathcal{E}^{2}$. This is a variation on a result in [16, Section 10.3]. Details may be found in [7] or [6, Section 3.6].

Theorem C.1. Suppose $\left\|\mathrm{e}^{A_{0} t}\right\| \leq \mathrm{e}^{\lambda t}$ for all $t \geq 0$. If there exists $\mu>\lambda$ such that

$$
(\lambda-\mu)^{2}>\left(\sum_{i=1}^{k}\left\|A_{i}\right\|\right)\left(\sum_{i=1}^{k}\left\|A_{i}\right\| \mathrm{e}^{-2 \mu \theta_{i}}\right),
$$

then there exists an equivalent inner product on $\mathcal{E}^{2}$ such that the delay semigroup $(T(t))$ satisfies

$$
\|T(t)\| \leq \mathrm{e}^{\mu t}, \quad t \geq 0 .
$$

By taking $\mu=0$, this theorem has the following corollary:

Corollary C.2. Suppose $\left\|\mathrm{e}^{A_{0} t}\right\| \leq \mathrm{e}^{\lambda t}$ for all $t \geq 0$. If

$$
\lambda<-\sum_{i=1}^{k}\left\|A_{i}\right\|
$$

then there exists an equivalent inner product on $\mathcal{E}^{2}$ such that the delay semigroup $(T(t))$ is a contraction semigroup, i.e. $\|T(t)\| \leq 1$ for all $t \geq 0$. 


\section{References}

[1] Appleby, J.A.D., Mao, X.: Stochastic stabilisation of functional differential equations. Syst. Control Lett. 54(11), 1069-1081 (2005)

[2] Arnold, L.: A formula connecting sample and moment stability of linear stochastic systems. SIAM J. Appl. Math. 44(4), 793-802 (1984)

[3] Arnold, L., Oeljeklaus, E., Pardoux, E.: Almost sure and moment stability for linear Itô equations. In: Lyapunov Exponents. Lecture Notes in Mathematics, vol. 1186, pp. 129-159 (1985)

[4] Arnold, L., Wihstutz, V.: Lyapunov exponents: a survey. In: Lyapunov Exponents (Bremen, 1984), Lecture Notes in Mathematics, vol. 1186, pp. 1-26. Springer, Berlin (1986)

[5] Bátkai, A., Piazzera, S.: Semigroups for Delay Equations. AK Peters, Ltd. (2005)

[6] Bierkens, J.: Long term dynamics of stochastic evolution equations. PhD thesis, Universiteit Leiden (2009)

[7] Bierkens, J.: Dissipativity of the delay semigroup. (2010, submitted)

[8] Bierkens, J., van Gaans, O., Verduyn Lunel, S.M.: Estimates on the pathwise Lyapunov exponent for linear stochastic differential equations with multiplicative noise. Stoch. Anal. Appl. 28(5), 747-762 (2010)

[9] Caraballo, T., Garrido-Atienza, M.J., Real, J.: Asymptotic stability of nonlinear stochastic evolution equations. Stoch. Anal. Appl. 21(2), 301-327 (2003)

[10] Caraballo, T., Liu, K., Mao, X.: On stabilization of partial differential equations by noise. Nagoya Math. J. 161, 155-170 (2001)

[11] Caraballo, T., Robinson, J.C.: Stabilisation of linear PDEs by Stratonovich noise. Syst. Control Lett. 53(1), 41-50 (2004)

[12] Cerrai, S.: Stabilization by noise for a class of stochastic reaction-diffusion equations. Probab. Theory Relat. Fields 133(2), 190-214 (2005)

[13] Curtain, R.F., Zwart, H.: An Introduction to Infinite-Dimensional Linear Systems Theory. Springer, New York (1995)

[14] Da Prato, G., Gatarek, D., Zabczyk, J.: Invariant measures for semilinear stochastic equations. Stoch. Anal. Appl. 10(4), 387-408 (1992)

[15] Da Prato, G., Zabczyk, J.: Stochastic Equations in Infinite Dimensions. Cambridge University Press, Cambridge (1992)

[16] Da Prato, G., Zabczyk, J.: Ergodicity for Infinite Dimensional Systems. Cambridge University Press, Cambridge (1996)

[17] Delfour, M.C.: The largest class of hereditary systems defining a $C_{0}$ semigroup on the product space. Can. J. Math. 32(4), 969-978 (1980)

[18] Engel, K.J., Nagel, R.: One-Parameter Semigroups for Linear Evolution Equations. Springer, New York (2000)

[19] Haussmann, U.G.: Asymptotic stability of the linear Itô equation in infinite dimensions. J. Math. Anal. Appl. 65(1), 219-235 (1978)

[20] Ichikawa, A.: Stability of semilinear stochastic evolution equations. J. Math. Anal. Appl. 90(1), 12-44 (1982)

[21] Kallianpur, G., Xiong, J.: Stochastic differential equations in infinite dimensional spaces. In: Lecture Notes-Monograph Series. Institute of Mathematical Statistics (1995) 
[22] Khasminskii, R.Z.: Stochastic stability of differential equations. In: Monographs and Textbooks on Mechanics of Solids and Fluids: Mechanics and Analysis, vol. 7. Sijthoff \& Noordhoff, Alphen aan den Rijn (1980). Translated from the Russian by D. Louvish

[23] Kwiecińska, A.A.: Stabilization of evolution equations by noise. Proc. Am. Math. Soc. 130(10), 3067-3074 (2002)

[24] Leizarowitz, A.: Exact results for the Lyapunov exponents of certain linear Ito systems. SIAM J. Appl. Math. 50(4), 1156-1165 (1990)

[25] Li, C.W., Dong, Z., Situ, R.: Almost sure stability of linear stochastic differential equations with jumps. Probab. Theory Relat. Fields 123(1), 121-155 (2002)

[26] Liu, K., Truman, A.: Moment and almost sure Lyapunov exponents of mild solutions of stochastic evolution equations with variable delays via approximation approaches. J. Math. Kyoto Univ. 41(4), 749-768 (2001)

[27] Liu, K., Truman, A.: Lyapunov function approaches and asymptotic stability of stochastic evolution equations in Hilbert spaces - a survey of recent developments. In: Stochastic Partial Differential Equations and Applications (Trento, 2002). Lecture Notes in Pure and Appl. Math., vol. 227, pp. 337-371. Dekker, New York (2002)

[28] Mao, X.: Robustness of exponential stability of stochastic differential delay equations. IEEE Trans. Automat. Control 41(3), 442-447 (1996)

[29] Mao, X.: Stochastic Differential Equations and Applications. Horwood, Chichester (1997)

[30] Mao, X., Shah, A.: Exponential stability of stochastic differential delay equations. Stoch. Stoch. Rep. 60(1-2), 135-153 (1997)

[31] Pardoux, É., Wihstutz, V.: Lyapunov exponent and rotation number of twodimensional linear stochastic systems with small diffusion. SIAM J. Appl. Math. 48(2), 442-457 (1988)

[32] Peszat, S., Zabczyk, J.: Stochastic partial differential equations with Lévy noise. In: Encyclopedia of Mathematics and its Applications, vol. 113. Cambridge University Press, Cambridge (2007)

[33] Scheutzow, M.: Stabilization and destabilization by noise in the plane. Stoch. Anal. Appl. 11(1), 97-113 (1993)

Joris Bierkens $(\bowtie)$

Centrum Wiskunde \& Informatica

P. O. Box 94079

1090 GB Amsterdam

The Netherlands

e-mail: j.bierkens@cwi.nl

Received: February 7, 2010.

Revised: October 25, 2010. 